\title{
CME Immunology and allergy (108458): self-assessment questionnaire
}

\section{Edited by Sarah Johnston and Tahseen A Chowdhury}

\section{SAQs and answers are ONLINE for RCP fellows and collegiate members}

SAQs and answers are ONLINE for RCP fellows and collegiate members

\section{Format}

Candidates are asked to choose the best answer from the five possible answers. This best of five format is used in many medical examinations, however the questions are not intended to be representative of those used in the MRCP(UK) Part 1 or Part 2 Written Examinations.

\section{The answering process}

1 Go to www.rcplondon.ac.uk/SAQ

2 Log on using your usual RCP username and password

3 Select the relevant CME question paper

4 Answer all 10 questions by selecting the best answer from the options provided

5 Once you have answered all the questions, click on Submit

\section{Registering your external CPD credits}

Carrying out this activity allows you to claim two external CPD credits. These will be automatically transferred to your CPD diary, where you can review the activity and claim your points.

1 Which of the following management strategies for allergic disease has been shown to influence the natural course of the disease?
(a) allergen avoidance
(b) allergen-specific immunotherapy
(c) antihistamines
(d) patient education
(e) topical steroids.

2 In the UK, allergen immunotherapy is used in the treatment of which of the following conditions?
(a) allergic rhinitis
(b) atopic dermatitis
(c) food allergy
(d) idiopathic anaphylaxis
(e) latex allergy.

3 Which of the following statements regarding immunoglobulin therapy is correct?

(a) According to Department of Health figures, expenditure on immunoglobulin $\mathrm{A}$ is less than $£ 100$ million annually.

(b) British plasma is used as a source of immunoglobulin production.

(c) Immunoglobulin can be prescribed for any autoimmune or inflammatory condition.

(d) The Demand Management Programme aims to secure immunoglobulin supplies for patients in greatest clinical need.

(e) There are no safety concerns related to the use of immunoglobulin.

4 Which of the following statements is correct regarding 'blue indications' for immunoglobulin?

(a) Alternative treatments should not be considered, even at times of immunoglobulin shortage, for patients with blue indication diseases.

(b) Blue indications are considered highest priority because of risk to life without treatment.

(c) Blue indications are divided into likely immune-mediated diseases with limited evidence of immunoglobulin efficacy and presumed immune-mediated diseases with little or no evidence of immunoglobulin efficacy.

(d) Blue indications are those conditions where there is evidence of benefit from immunoglobulin but for which there may be alternative treatment options.

(e) Blue indications have no evidence base on which to support immunoglobulin treatment.

5 A 62-year-old male patient presented with recurrent episodes of feeling faint, associated with flushing, abdominal cramps and diarrhoea. The patient had been rushed to the emergency department with an anaphylactic reaction to a wasp sting 6 months earlier. On examination, there was no visible rash and no palpable organomegally. A full blood count was unremarkable. You suspect a mast cell activation disorder. What would be the most appropriate next investigation?
(a) bone marrow biopsy
(b) $\mathrm{CT}$ chest, abdomen, pelvis
(c) screening for a D816V KIT mutation of an EDTA blood sample[c1]
(d) serum tryptase
(e) specific IgE to wasp venom. 
6 A 45-year-old female patient presented with red brown macules increasing in number over the abdomen and thighs over the preceding 6 months. The patient noted that when the rash was rubbed, some of the lesions became raised and itchy. She was otherwise well with no associated symptoms, such as flushing or diarrhoea. There was no history of anaphylaxis. A skin biopsy was performed and a diagnosis of urticaria pigmentosa was confirmed. Serum tryptase was $<14 \mathrm{ng} / \mathrm{mL}$. Which of the following treatments are likely to benefit the patient at this stage?
(a) antihistamines
(b) dairy free diet
(c) hydrocortisone ointment
(d) imatinib
(e) sodium cromoglicate.

7 Skin prick testing is generally helpful for the diagnosis of:
(a) allergy to nonsteroidal anti-inflammatory drugs
(b) angiotensin-converting enzyme inhibitor angioedema
(c) opiate reactions
(d) penicillin allergy
(e) Stevens-Johnson syndrome.

8 Which of the following is correct about angiotensinconverting enzyme (ACE) inhibitor angioedema?

(a) Angiotensin receptor blockers must not be tried.

(b) Episodes always stop immediately following discontinuation of the ACE inhibitor.

(c) Episodes can happen several years after starting the ACE inhibitor. (d) Patients should be referred for specialist review.

(e) The ACE inhibitor should be continued with addition of antihistamine prophylaxis.

9 Clinically significant secondary antibody deficiency:
(a) always occurs with B-cell depleting therapies
(b) can be managed with prophylactic antibiotic therapy
(c) can lead to silent end-organ damage, eg bronchiectasis
(d) typically presents with recurrent infections with staphylococcus
(e) usually occurs in solid organ malignancies.

10 Rituximab therapy-induced hypogammaglobulinaemia
(a) depletes plasma cells, resulting in loss of antibody production
(b) is managed with prophylactic antibiotic therapy
(c) is more likely with multiple cycles of therapy
(d) is significant in the majority of patients treated with the drug
(e) is usually permanent.

\section{CME Clinical pharmacology SAQ Answers to the CME SAQ published in Clinical Medicine in October 2016}
Q1 Q2 Q3
$\begin{array}{ll}\text { Q4 } & \text { Q5 } \\ \text { (b) } & \text { (b) }\end{array}$
Q6
(a) (d) (a)
(e) (e)
Q7 Q8
Q8 Q9
Q10
(d) 